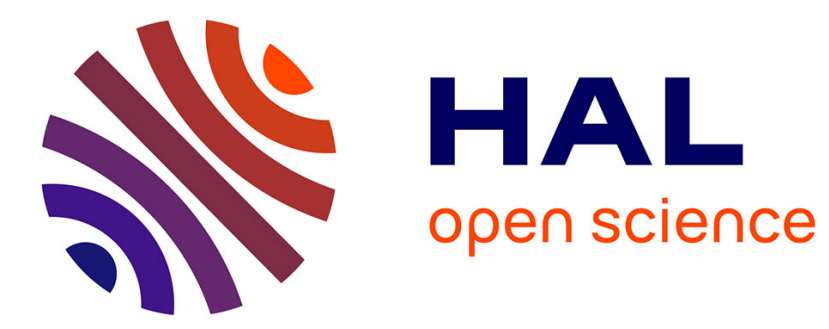

\title{
Emploi de capteurs solaires cylindro-paraboliques pour la réfrigération dans les zones tropicales
}

\author{
J. Fléchon, A. Kotowski, F. Machizaud, G. Godmel
}

\section{To cite this version:}

J. Fléchon, A. Kotowski, F. Machizaud, G. Godmel. Emploi de capteurs solaires cylindro-paraboliques pour la réfrigération dans les zones tropicales. Revue de Physique Appliquée, 1982, 17 (9), pp.577-584. 10.1051/rphysap:01982001709057700 . jpa-00245034

\section{HAL Id: jpa-00245034 https://hal.science/jpa-00245034}

Submitted on 1 Jan 1982

HAL is a multi-disciplinary open access archive for the deposit and dissemination of scientific research documents, whether they are published or not. The documents may come from teaching and research institutions in France or abroad, or from public or private research centers.
L'archive ouverte pluridisciplinaire HAL, est destinée au dépôt et à la diffusion de documents scientifiques de niveau recherche, publiés ou non, émanant des établissements d'enseignement et de recherche français ou étrangers, des laboratoires publics ou privés. 
Classification

Physics Abstracts

75.60

\title{
Emploi de capteurs solaires cylindro-paraboliques pour la réfrigération dans les zones tropicales
}

\author{
J. Fléchon, A. Kotowski, F. Machizaud et G. Godmel \\ Laboratoire de Physique des Dépôts Métalliques, Université de Nancy I, \\ Case Officielle 140, 54037 Nancy cedex, France
}

(Reçu le 26 octobre 1981, révisé le 12 mars 1982, accepté le 22 avril 1982)

\begin{abstract}
Résumé. - La réfrigération obtenue par photothermie exige au générateur des températures supérieures à $100{ }^{\circ} \mathrm{C}$ seules susceptibles de dissocier le couple frigorigène avec un rendement acceptable. Dans ce but, nous étudions les conditions et les effets d'un captage de rayonnement à sous-concentration.
\end{abstract}

\begin{abstract}
The refrigeration obtained by photothermy requires in the generator temperatures greater than $100^{\circ} \mathrm{C}$, the lower limit for dissociating the frigorigene couple with an acceptable efficiency. In this aim, we have studied half concentration captage of radiation conditions and effects.
\end{abstract}

Nous avons étudié des capteurs solaires de forme cylindro-parabolique à semi-concentration (coefficient de concentration 5).

Dans les réfrigérateurs solaires, ces capteurs jouent le rôle de générateurs. Ils comportent : une enveloppe isolante, un tube focal absorbant, une surface réfléchissante cylindro-parabolique ainsi qu'une ou plusieurs vitres protégeant le capteur des fuites thermiques liées à la température ambiante.

Nous avons retenu, pour la réfrigération, la concentration dans le but de pouvoir atteindre au générateur, une température suffisamment élevée assurant la dissociation du couple contenu dans le tube focal et libérant le frigorigène en phase gazeuse. Mais un certain nombre d'exigences contradictoires ont été à l'origine d'une optimisation du capteur. En effet, diminuer le diamètre du tube focal permet d'atteindre rapidement des températures suffisantes mais entraîne deux inconvénients majeurs : une forte directivité du récepteur ce qui provoque de fréquentes réorientations de l'appareil et la réduction de la quantité de matière formant le couple frigorigène ce qui diminue son rendement.

Différentes surfaces de miroirs sont simultanément utilisées au sein d'une même enveloppe afin de maintenir comparables les conditions d'utilisation du capteur. Sur un bâti métallique de forme cylindro-parabolique, on applique des feuilles constituées des matériaux suivants : inox, aluminium, plastique argent, plastique cuivre, soit 4 capteurs en parallèle.
Le tube focal en acier présente une surface absorbante externe, recouverte de peinture noir-mat ou d'une feuille mince sélective. Il contient de l'huile dont on enregistre la température. L'énergie incidente mesurée à l'aide d'une cellule photovoltaique étalon est également enregistrée. Le nombre de vitres varie de 0 à 3 , leur distance de 2 à $20 \mathrm{~mm}$.

On examine l'influence sur :

- la température d'équilibre,

- le rendement énergétique,

- les pertes énergétiques,

- la cinétique d'élévation de température.

des différents paramètres indiqués plus haut.

Les jours où l'ensoleillement le permet, nous exposons le capteur à l'extérieur et travaillons ainsi sur le rayonnement direct du soleil à Nancy. Les autres jours, nous utilisons un simulateur à lampes.

Nous discutons les résultats et obtenons au soleil, par jour clair, en mai 1981, dans les meilleures conditions de construction, une température d'équilibre largement supérieure à $100^{\circ} \mathrm{C}$.

1. Méthodes et techniques expérimentales. -1.1 Appareillage. - Afin de nous placer dans des conditions expérimentales reproductibles, nous avons disposé 4 capteurs linéaires cylindro-paraboliques $(L \times l) L=30 \mathrm{~cm}, l=25 \mathrm{~cm}$ sur une même surface de $\left(L \times l \mathrm{~m}^{2}\right)$ isolés thermiquement les uns des autres (Fig. 1). Chaque capteur possède un tube cylindrique 
en acier de diamètre $50 \mathrm{~mm}$ (absorbeur) contenant $500 \mathrm{~cm}^{3}$ d'huile suivant l'axe duquel est glissé un thermocouple (NiCr-Ni allié), le tube est recouvert d'une couche absorbante et disposé suivant l'axe focal. Les dimensions du réflecteur et de l'absorbeur sont telles que le coefficient de concentration (surface du capteur/ surface apparente de l'absorbeur) soit égal à 5 . Une photopile positionnée au centre de la surface exposée

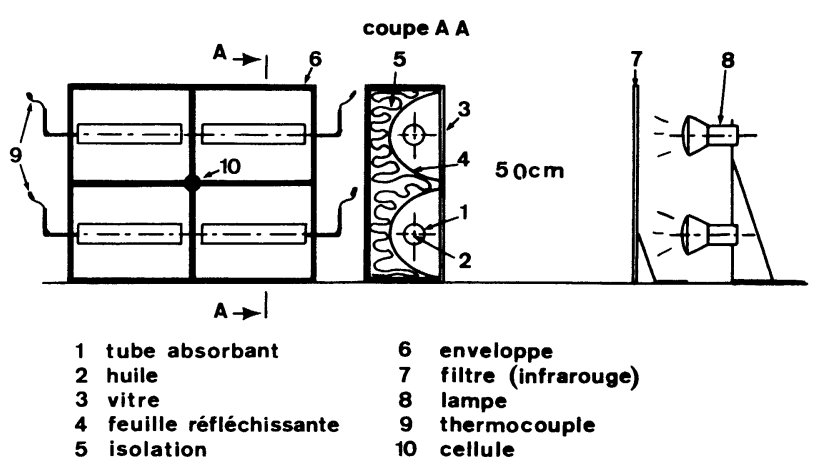

Fig. 1. - Appareil pour l'étude avec simulateur.

[Apparatus for study with simulator.]

au rayonnement permet de mesurer la puissance incidente. L'étude peut être faite en utilisant soit le rayonnement solaire, soit une simulation en laboratoire en éclairant normalement la surface des 4 capteurs par 4 lampes (P.A.R. 38, Philips, $150 \mathrm{~W}$ ) placées à environ $50 \mathrm{~cm}$ de ceux-ci. Dans le premier cas, la surface des capteurs est orientée au Sud et inclinée de façon que le rayonnement solaire tombe normalement sur elle à 12 h. T.S.V. Dans le 2e cas, afin d'atténuer le rayonnement infrarouge, nous interposons un vitrage de $4 \mathrm{~mm}$ entre capteurs et lampes (Fig. 1).

Il est ainsi possible d'étudier simultanément et pour une même énergie incidente, l'influence des paramètres caractérisant le capteur. Ce sont :

1) distance entre vitres $(2 \mathrm{~mm}, 7 \mathrm{~mm}, 13 \mathrm{~mm}$, $20 \mathrm{~mm}$ ) pour un même matériau réflecteur et une même couche absorbante (couche de peinture noire mate non sélective);

2) nombre de vitres $(0,1,2,3)$;

3) nature des 4 matériaux réflecteurs que nous avons retenus : plastique argenté, plastique cuivré, feuille d'aluminium $(1,5 \mathrm{~mm})$ feuille d'inox $(1 \mathrm{~mm})$;

4) nature de la couche absorbante (peinture noire mate non sélective, feuille de Maxorb à surface sélective), pour un même matériau réflecteur (plastique argenté).

1.2 TeChNique De Mesure. - Sont déterminées simultanément et en continu sur un enregistreur MECI :

- la température ambiante,
- la température de l'huile dans chacun des quatre capteurs,

- la puissance du rayonnement incident.

2. Traitement mathématique des données (Fig. 2). L'éclairement énergétique incident $J_{\mathrm{m}} \mathrm{W} / \mathrm{m}^{2}$ est calculé en faisant la moyenne des $n$ mesures prises sur $1 / 2$ heure,

$$
J_{\mathrm{m}}=\frac{\sum_{1}^{n} J_{\mathrm{i}}}{n}
$$

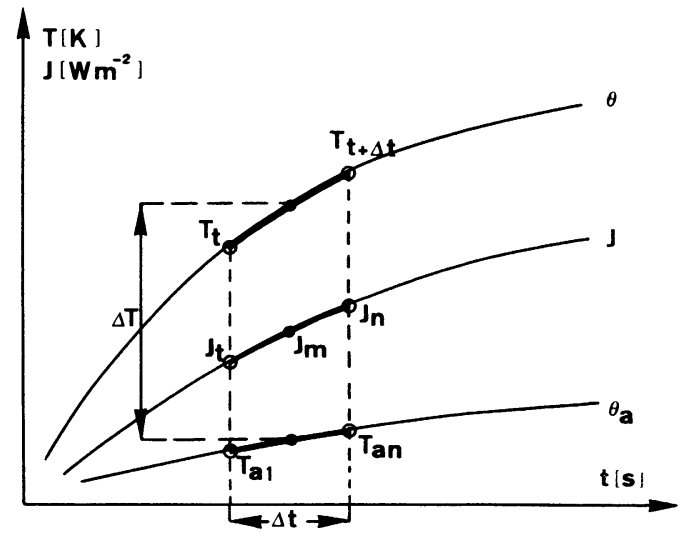

Fig. 2. - Principe du calcul.

[Principle of the calculation.]

ce qui nous permet de connaître la puissance reçue $J_{\mathrm{c}}$ pour un capteur de surface $A$

$$
J_{\mathrm{c}}=J_{\mathrm{m}} \cdot A \quad\left(A=0,07 \mathrm{~m}^{2}\right) .
$$

Afin d'accéder au rendement d'un capteur, la puissance utilisable par celui-ci est définie par la relation :

$$
\begin{aligned}
J_{\mathrm{u}}= & \frac{V \cdot \rho \cdot c\left(T_{t+\Delta t}-T_{t}\right)}{\Delta t} \\
V & =0,005 \mathrm{~m}^{3} \\
\rho & =900 \mathrm{~kg} / \mathrm{m}^{3} \\
c & =1920 \mathrm{~J} / \mathrm{kg} \cdot \mathrm{K} \\
\Delta t & =1800 \mathrm{~s} .
\end{aligned}
$$

$V=$ volume d'huile, $c=$ chaleur massique de l'huile, $\rho=$ densité de l'huile, $\left(T_{t+\Delta t}-T_{t}\right)$ variation de température correspondant à un intervalle de temps $\Delta t$.

Soit pour le rendement à l'instant $t$ :

$$
R=\frac{J_{\mathrm{u}}}{J_{\mathrm{c}}}
$$

et pour les pertes instantanées :

$$
P=1-R \text {. }
$$


Les pertes instantanées dépendent fortement de l'écart entre la température $T$ de l'huile et celle $T_{\mathrm{a}}$ de l'air ambiant. Nous calculons un écart moyen défini par la relation :

$$
\Delta T=\frac{\sum_{1}^{n} T_{\mathrm{i}}}{n}-\frac{\sum_{1}^{n} T_{\mathrm{ai}}}{n}
$$

la moyenne de mesures étant faite sur $1 / 2$ heure. Les résultats expérimentaux seront présentés sous deux formes :

$$
R=f\left(\frac{\Delta T}{J_{\mathrm{c}}}\right)
$$

$$
P=G\left(\frac{\Delta T}{J_{\mathrm{c}}}\right)
$$

Précisons l'intérêt de cette formulation en introduisant le rapport $\Delta T / \Delta T$

$$
R=\frac{J_{\mathrm{u}}}{J_{\mathrm{c}}}=\frac{V \rho c \delta T \Delta T}{\delta t J_{\mathrm{c}} \Delta T}
$$

$\delta T$ : élévation de température du bain par unité de temps,

$\Delta T$ : écart à l'ambiante du bain.

Soit : $x=\Delta T / J_{\mathrm{c}} \rho, V, c$ étant constants. $R$ s'écrit en tenant compte de l'expérience.

$$
R=K \frac{\delta T}{\delta t \Delta T} x=-a x+b=x\left(\frac{b}{x}-a\right)
$$

d'où :

$$
K \frac{\delta T}{\delta t}=b J_{\mathrm{c}}-a \Delta T
$$

ce qui revient à dire que :

$$
J_{\mathrm{c}}=\frac{K \delta T}{b \delta t}+\frac{a}{b} \Delta T
$$

équation qui traduit un phénomène de conservation de l'énergie. L'énergie reçue est une fonction linéaire de l'élévation de température du capteur par unité de temps et de l'écart à l'ambiante de la température du capteur.

Ceci justifie la représentation retenue : le rendement du capteur est une fonction linéaire de la variable définie par le quotient de l'écart à l'ambiante du caloporteur par l'intensité du rayonnement incident.

Nous obtenons de cette manière un diagramme simple et directement exploitable. Notons que les fuites thermiques apparaissent dans l'équation (1) en déterminant les coefficients $a$ et $b$ après avoir retranché $J_{\mathbf{u}}$.

3. Résultats expérimentaux. - 3.1 EXPÉRIENCE AVEC SIMULATION : $J_{\mathrm{c}}=40 \mathrm{~W}, J_{\mathrm{m}}=600 \mathrm{~W} \mathrm{~m}^{-2}$. 3.1.1 Distance entre vitres. - L'expérience a été conduite avec deux puis trois vitres (Figs. 3 et 4). Le rendement optimal est réalisé dans les deux cas pour une séparation entre vitres de $15 \mathrm{~mm}$. L'examen du diagramme montre en effet que pour deux vitres très rapprochées tout se passe comme si on n'utilisait qu'une seule vitre très épaisse. Trop éloignées, elles n'assurent plus une bonne isolation de l'enceinte tout en en réduisant l'intensité du rayonnement incident. Le volume d'air emprisonné entre les deux vitres est optimal pour une distance d'environ $15 \mathrm{~mm}$.

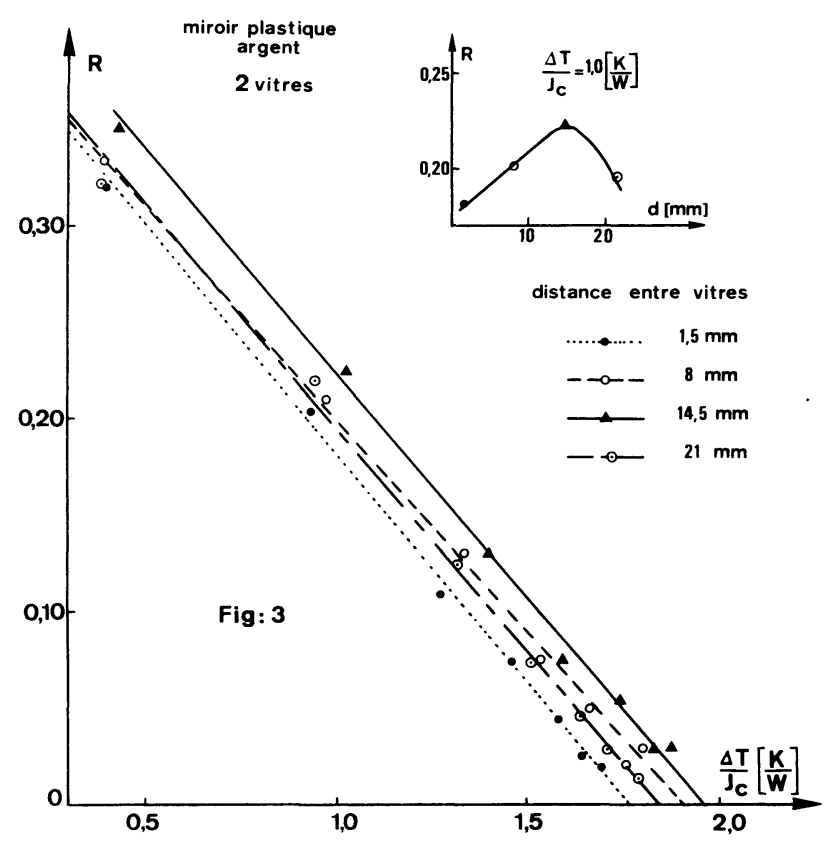

Fig. 3. - Rendement en simulation : 2 vitres à différentes distances.

[Efficiency in simulation : 2 glasses to variable distances.]

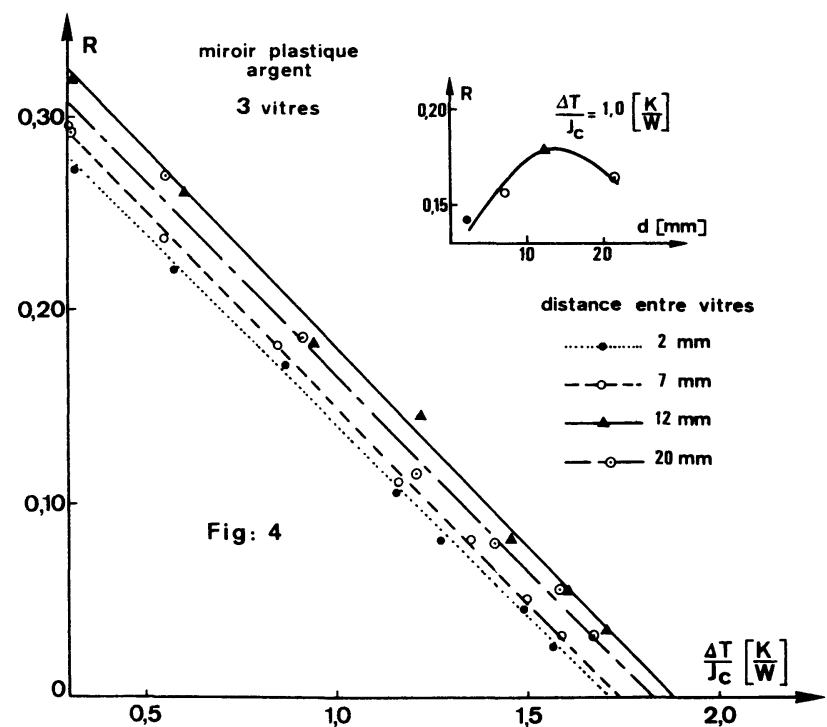

Fig. 4. - Cas de 3 vitres.

[Case of 3 glasses.] 
3.1.2 Le nombre de vitres. - La distance entre vitres est fixée à $15 \mathrm{~mm}$ d'après les résultats du $\S 3.1 .1$.

3.1.2.1 Influence sur le rendement. - La figure 5 résume les résultats et permet les remarques suivantes : le rendement obtenu avec 0 vitre chute rapidement dès que le capteur s'échauffe, celui obtenu avec 3 vitres demeure généralement inférieur à celui réalisé avec 1 ou 2 vitres.

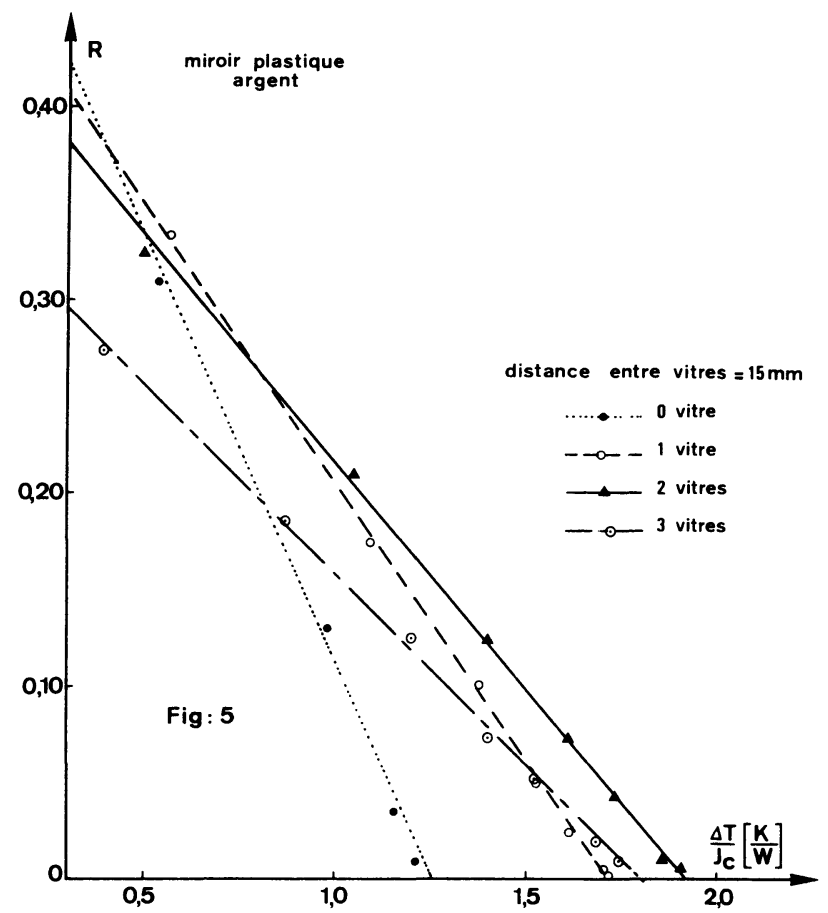

Fig. 5. - Rendement en fonction du nombre de vitres et de $x=\Delta T / J_{c}$.

[Efficiency versus number of glasses and $x=\Delta T / J_{\mathrm{c}}$.]

Le dispositif avec 0 vitre reste cependant le mieux adapté pour $\Delta T / J_{\mathrm{c}}<0,4 \mathrm{~K} / \mathrm{W}$.

Pour $0,4<\Delta T / J_{c}<0,8$, le meilleur rendement est obtenu avec 1 vitre. Lorsque $\Delta T / J_{c}>0,8$, il apparaît préférable d'utiliser un capteur muni de 2 vitres.

D'après les études précédentes sur les capteurs plans (1) (2) (3) (4) deux variables essentielles doivent être définies :

- la cinétique d'échauffement sous rayonnement d'intensité fixe,

- la température limite atteinte dans ces conditions par le capteur au point de stagnation pour lequel $R=0$.

L'intérêt de la figure 5 est de permettre la détermination directe de la cinétique par la lecture des pentes des droites, l'échauffement sous 0 vitre est plus rapide que celui sous 1 puis 2 puis 3 , ce qui confirme la théorie (Fig. 5).

De même $R=0$ nous permet de définir la température limite dans les conditions de l'expérience soit :
$T 51 \mathrm{~K}$ avec 0 vitre atteint en 2,1 heures

$\begin{array}{lll}68 \mathrm{~K} & 1 \text { vitre } & 3,3 \\ 76 \mathrm{~K} & 2 \text { vitres } & 4,2 \\ 72 \mathrm{~K} & 3 \text { vitres } & 4,9\end{array}$

Il semble qu'au-delà de deux vitres, la théorie soit en défaut. En réalité, l'échauffement est plus lent et les fuites thermiques l'emportent dès que diminue le gradient $\delta T / \delta t$.

Pratiquement pour la réfrigération où nous recherchons une température élevée l'emploi de deux vitres semble le mieux adapté.

3.1.2.2 Les pertes. - Les mêmes conclusions permettant de définir les meilleures conditions expérimentales peuvent être déduites de la figure 6 donnant les pertes en fonction de $\Delta T / J_{\mathrm{c}}$. Celles-ci sont, comme l'on sait dues à la convection, la conduction et le rayonnement.

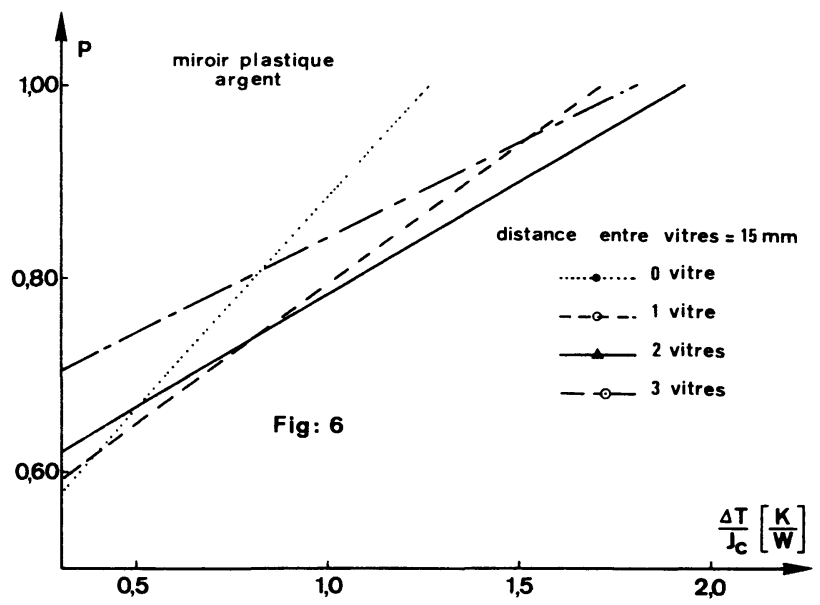

Fig. 6. - Pertes dans les mêmes conditions.

[Losses in the same conditions.]

L'avantage de la représentation $R=f(x)$ qui a permis le tracé d'un diagramme linéaire apparaît tout naturellement dans l'expression des pertes $P=1-R$ qui doivent s'exprimer linéairement en fonction de $x$. C'est bien ce que l'on observe sur la figure 6. Les pentes des droites s'ordonnent comme précédemment : les fuites thermiques les plus fortes se produisant pour 0 vitre, les plus faibles pour 3 .

3.1.2.3 Cinétique d'élévation de la température. La relation liant le temps à $x$ est logarithmique. La figure 7 conduit à des conclusions semblables en portant $\log R$ en fonction de $\log t$. Le maximum d'élévation de température étant atteint au bout du temps $t$ en heures a été indiqué dans le tableau précédent. Si l'on tient compte de la brièveté de la durée de l'insolation efficace $(6 \mathrm{~h}$ dans les conditions les meilleures assurant la limpidité du ciel) on observe qu'un compromis est nécessaire entre la rapidité de l'échauffement et la hauteur du point de stagnation. 


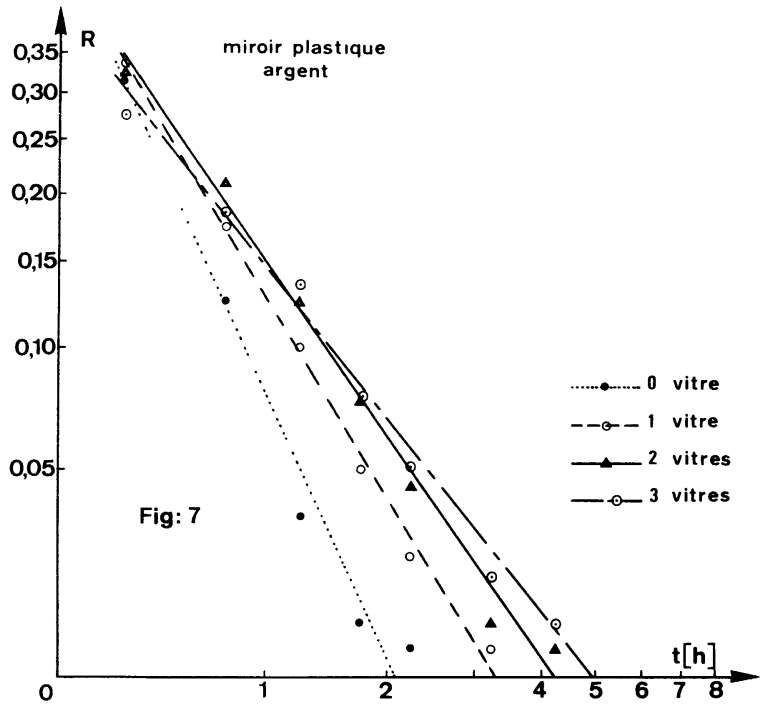

Fig. 7. - Rendement en fonction du temps.

[Efficiency versus time.]

3.1.3 Nature du matériau réflecteur. - Cette étude a été menée dans le cas de capteurs possédant respectivement 0 vitre, 1 vitre et 2 vitres (Figs. 8, 9, 10). Afin de rendre plus facile l'interprétation de ces résultats expérimentaux, nous représentons sur la figure 11, le rendement obtenu par chaque type de réflecteur pour $\Delta T / J_{\mathrm{c}}=1,0$ et pour $0,1,2$ vitres.

Il semble ressortir de cette expérience que la qualité du réflecteur s'accroît lorsque l'on passe de l'inox, à l'aluminium, au plastique argenté puis au plastique cuivré.

Notons cependant que la différence de rendement

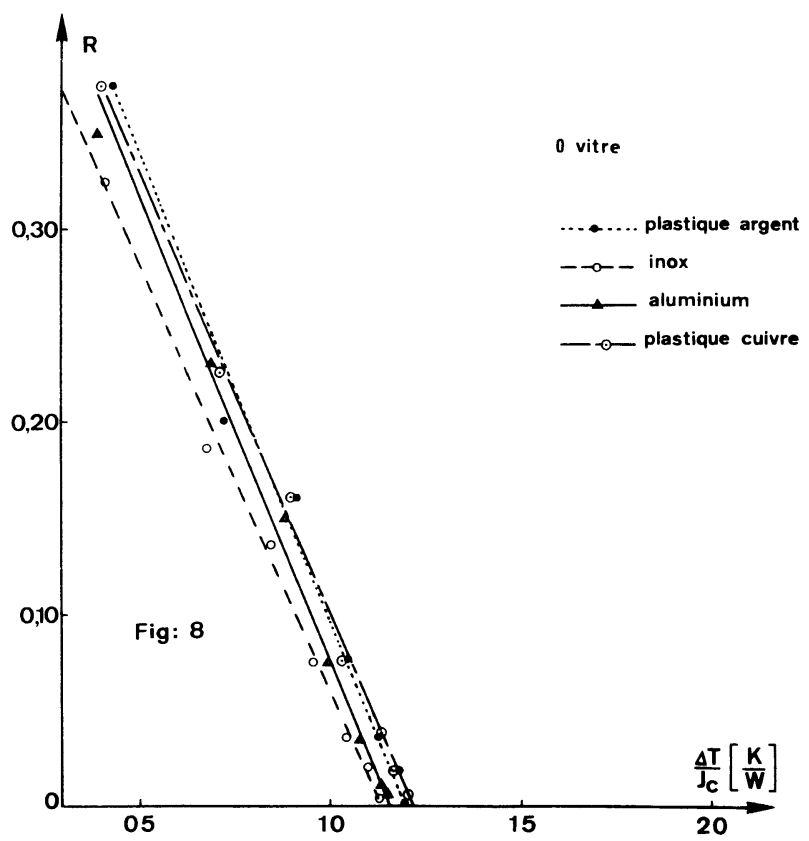

Fig. 8. - Rendement avec différents réflecteurs 0 vitre.

[Efficiency with different reflectors 0 glass.]

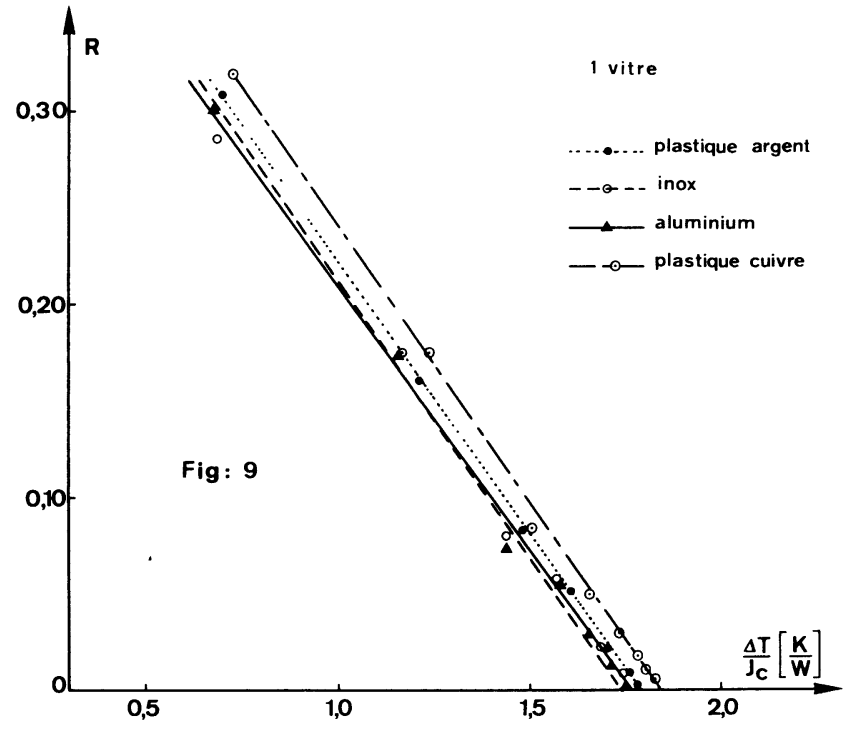

Fig. 9. - Cas de 1 vitre.

[Case of 1 glass.]

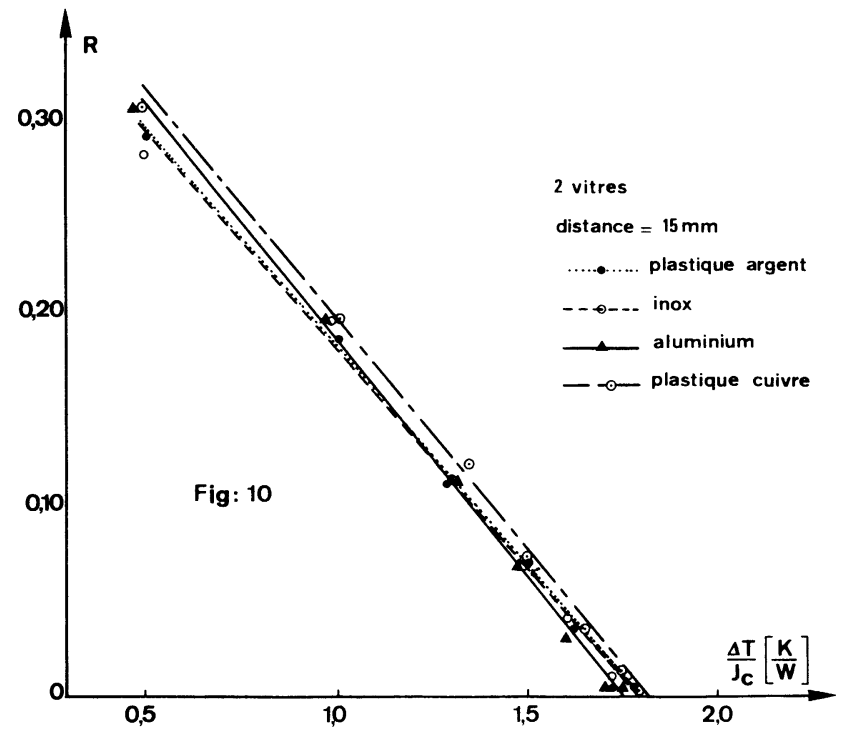

Fig. 10. - Cas de 2 vitres.

[Case of 2 glasses.]

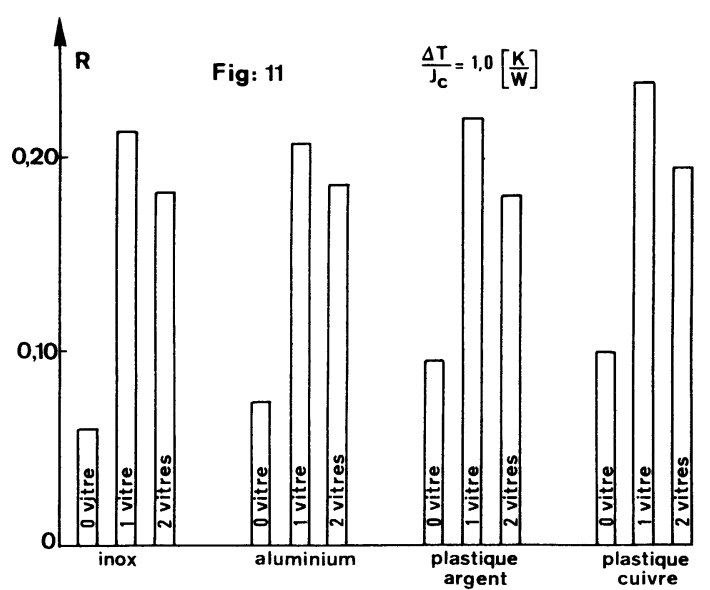

Fig. 11. - Comparaison des rendements simulés à $x$ donné [Giving $x$ comparison of the efficiencies in simulation.] 
entre un réflecteur en plastique cuivré et un réflecteur en inox est inférieure à $4 \%$ ce qui est important pour la réalisation ultérieure des capteurs.

3.1.4 Sélectivité de la surface absorbante. - Nous avons utilisé comme surface non sélective de l'absorbeur une couche de peinture noir mat (600 g d'oxyde de fer noir; $350 \mathrm{~g}$ d'éburit cocket rouge), $500 \mathrm{~g}$ de White Spirit dont nous avons comparé les résultats enregistrés à ceux obtenus avec une surface sélective constituée par une feuille de "Maxorb".

La figure 12 consigne les résultats :

- quel que soit le nombre de vitres $(0,1,2)$ la surface sélective donne toujours le meilleur rendement,

- des expériences précédentes réalisées avec une couche non sélective $(\S 3.1 .2)$ nous avons remarqué que les conditions optimales étaient réalisées avec un double vitrage pour $\Delta T / J_{\mathrm{c}}=0,8$.

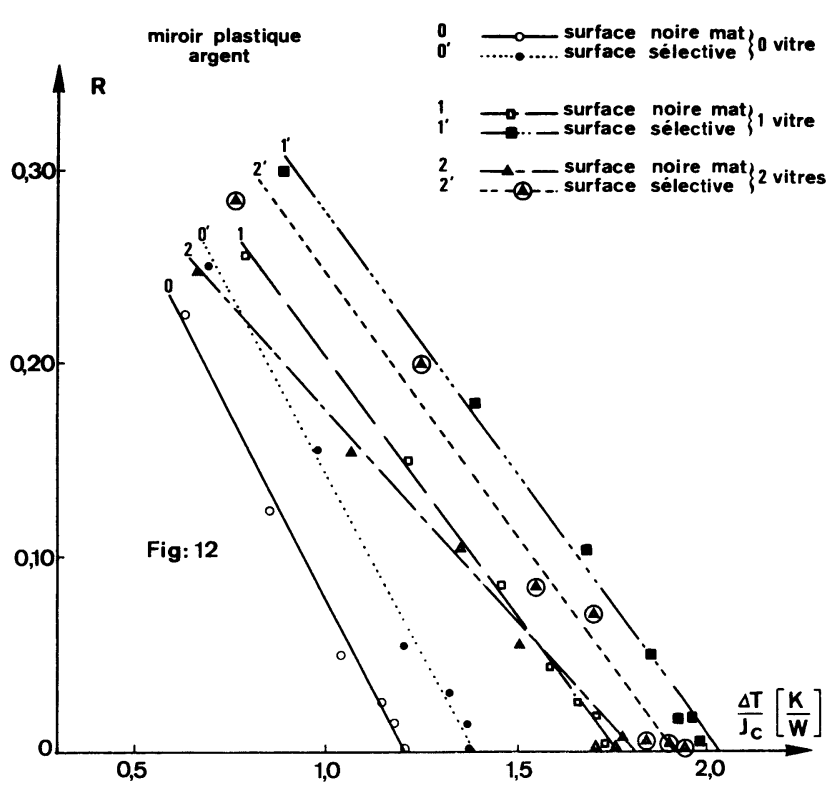

Fig. 12. - Surface sélective et corps noir.

[Selective area and black body.]

Il apparait ici dans le cas de l'utilisation d'une couche sélective qu'une seule vitre suffit pour nous placer dans les meilleures conditions. Ceci s'explique par le fait que la surface sélective est absorbante dans le visible et faiblement émettrice dans l'infrarouge.

\subsection{EXPÉRIENCES SOUS RAYONNEMENT SOLAIRE. -} Il était important d'examiner le comportement réel des capteurs sous irradiation solaire. Ce genre d'essai se heurte à de nombreux obstacles surtout sous le climat lorrain. On ne peut en effet travailler que sous rayonnement direct, donc en ciel clair mais en l'absence totale de nuages car une occultation, même brève, des rayons solaires, laisse jouer les seules fuites thermiques et perturbe les résultats. Cependant, la difficulté de reproduire la distribution spectrale énergé-

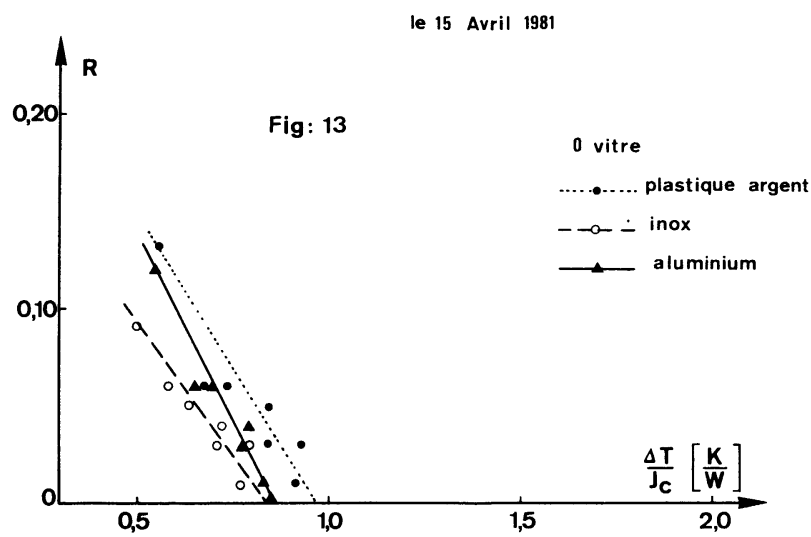

Fig. 13. - Etude au soleil avec différents réflecteurs 0 vitre. [Solar study with different reflectors 0 glass.]

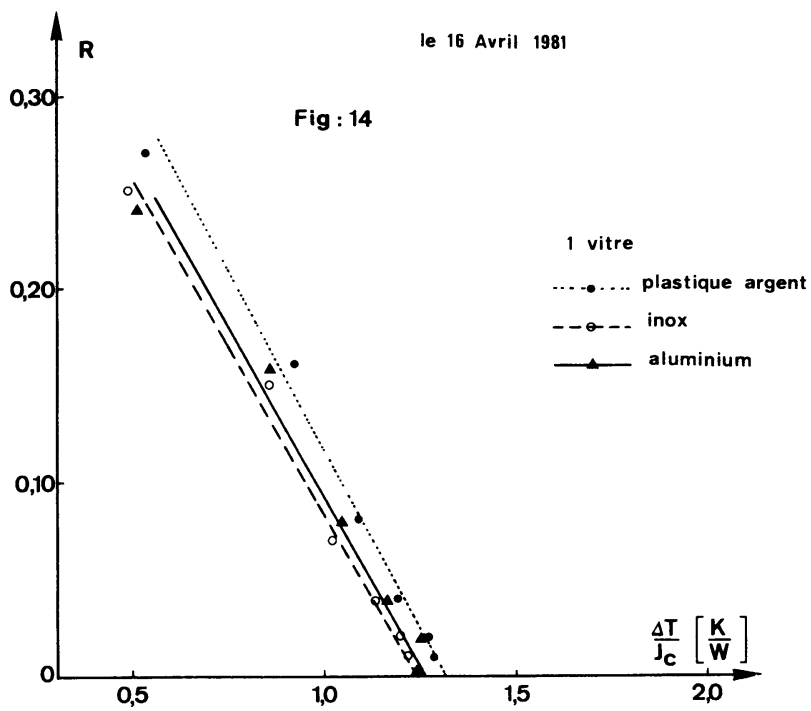

Fig. 14. - Cas d'une vitre. [Case of a glass.]

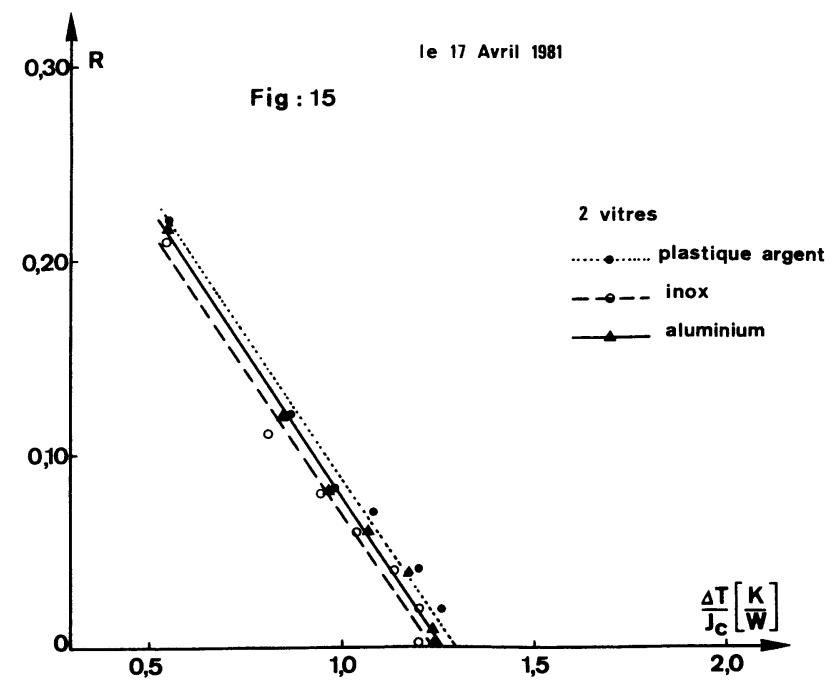

Fig. 15. - Cas de 2 vitres.

[Case of 2 glasses.] 


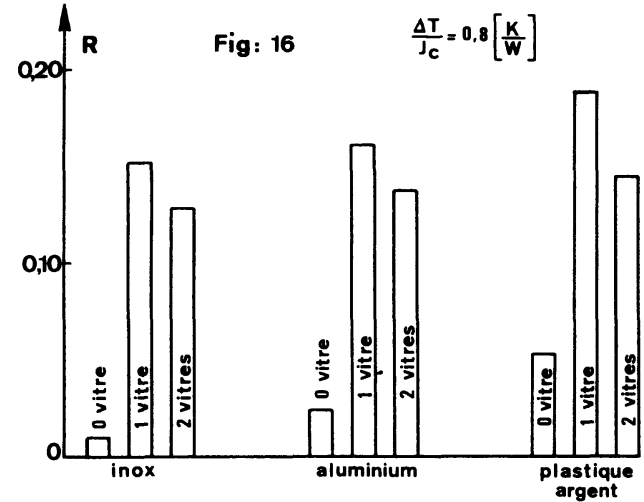

Fig. 16. - Comparaison des rendements au soleil à $x$ donné.

[Giving $x$ comparison of the efficiencies in solar radiations.]

tique du direct rend nécessaire le test au soleil. Nous avons donc attendu les jours clairs pour tenter des essais comparatifs en reprenant l'examen des paramètres vus en simulation. Ce sont :

3.2.1 Nature du réflecteur. - Les figures $13,14,15$ et 16 nous amènent aux mêmes conclusions en ce qui concerne le moins bon réflecteur, c'est-à-dire l'inox, par contre le meilleur réflecteur est ici le plastique argenté et non le plastique cuivré, la différence de rendement demeurant toujours faible.

3.2.2 Nature de labsorbeur et nombre de vitres. Deux expériences successives ont été conduites dans des conditions analogues malgré l'évidente difficulté que représente toute expérience conduite au soleil en vraie grandeur. L'état du ciel doit être aussi voisin que possible dans les deux cas et les iours retenus ne doivent pas être trop éloignés pour que le rayonnement direct n'évolue pas trop d'une expérience à l'autre.

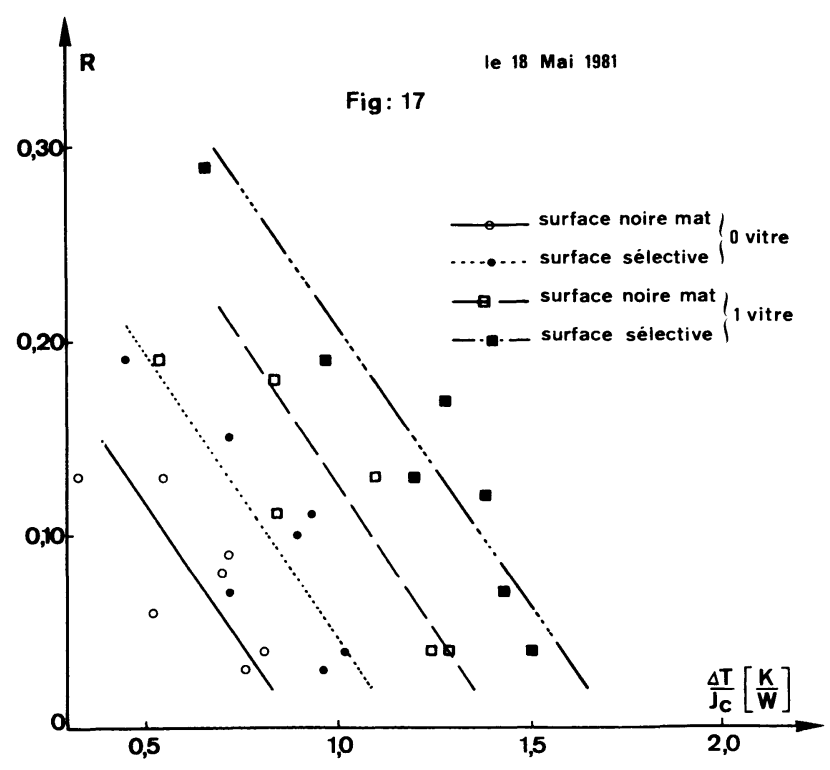

Fig. 17. - Surface sélective et corps noir au soleil (0-1 vitre).

[Selective area and black body in solar radiation (0-1 glass).]

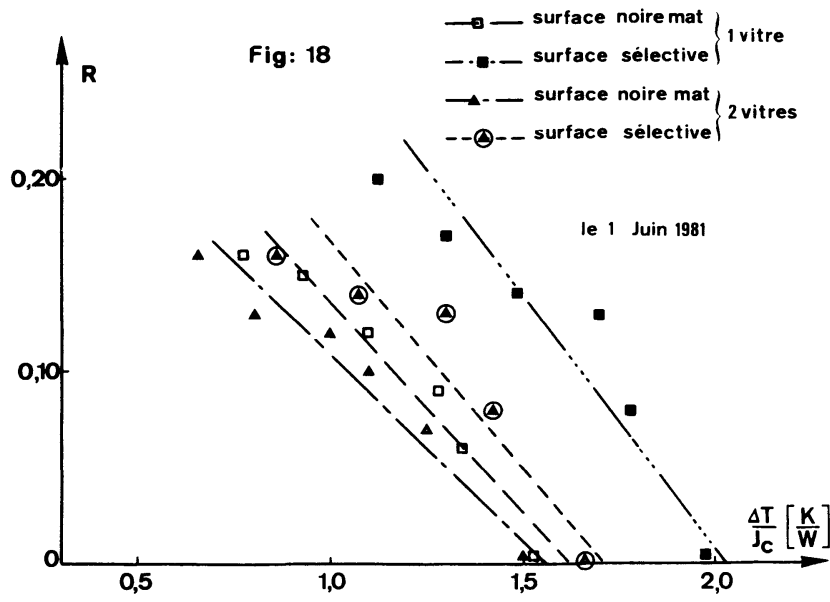

Fig. 18. - Cas de 1 et 2 vitres au sole1l.

[Case of 1 and 2 glasses in solar radiation.]

Nous avons éliminé les essais avec trois vitres étant donnée l'insuffisance des performances obtenues. $\mathrm{La}$ comparaison porte donc sur 0-1 et 1-2 vitres.

\begin{tabular}{|c|c|c|c|}
\hline & No & Expérience 1 & Expérience 2 \\
\hline Absorbeur & Capteur & vitres & vitres \\
\hline \multirow{3}{*}{ noir mat } & - & - & - \\
\hline & 1 & 0 & 1 \\
\hline & 2 & 1 & 2 \\
\hline «maxorb & 3 & 0 & 1 \\
\hline sélectif » & 4 & 1 & 2 \\
\hline
\end{tabular}

Les résultats relatifs à ces deux expériences (Figs. 17, 18) confirment ceux du paragraphe 3.1 .4 , la surface sélective est indispensable pour obtenir le meilleur rendement que le capteur ait 0,1 ou 2 vitres.

L'ordre d'efficacité décroissante des capteurs est identique :

1) 1 vitre surface sélective,

2) 2 vitres surface sélective,

3) 1 vitre surface non sélective,

4) 2 vitres surface non sélective,

5) 0 vitre surface sélective,

6) 0 vitre surface non sélective

Observons que sous rayonnement direct la source est à l'infini. Dans ce cas, l'image obtenue sur le récepteur est différente de celle résultant de la simulation puisque la source émettrice de rayonnement est un ensemble de lampes à incandescence munies de réflecteurs et situées à $50 \mathrm{~cm}$ environ du capteur. Or les réflecteurs des lampes orientent le faisceau incident dans une direction sensiblement axiale et peu ouverte - pratiquement ces lampes sont appelées "spots" ce qui souligne leur directivité. La différence entre la simulation et l'expérience réelle semble d'autant moins importante que le diamétre du tube focal est le $1 / 5$ de l'ouverture du miroir. 
Nous avons initialement retenu ce rapport en raison $\mathrm{du}$ but poursuivi : obtenir en zone tropicale une température suffisamment élevée pour assurer, durant les 6 heures d'insolation efficace, la dissociation de l'absorbat solide placé dans le tube focal. Notons également que l'avantage du cylindro-parabolique est réduit par son immobilité mais cette recherche se voulant une étape de la réalisation d'un réfrigérateur solaire, cette contrainte nous est imposée par la destination de l'appareil si nous ne voulons pas annoncer des résultats qui seront démentis par une expérience réelle. L'usager présumé ne devant jamais intervenir sauf pour nettoyer les vitres et modifier quatre fois par an la pente du plan récepteur.

4. Conclusion. - Une comparaison entre les rendements obtenus en simulation et au soleil montre que le rendement, essentiellement variable en fonction de la température du capteur, atteint dans le premier cas un maximum moyen de $25 \%$ et de $20 \%$ dans le second. Le rendement du capteur exposé au soleil serait plus faible qu'en simulation. Ceci est explicable par le fait, qu'au soleil, le capteur est exposé au vent et les fuites thermiques sont, dans ces conditions, très supérieures à celles qui existent dans l'air au repos d'un laboratoire. Nous avons montré dans le calcul du point de stagnation (3) et en réfrigération solaire l'importance capitale des fuites thermiques (5). D'autre part, la mesure des rayonnements est effectuée à l'aide d'une photopile étalonnée à l'usine R.T.C. de Caen par l'emploi d'un arc au xénon. La distribution spectrale énergétique du rayonnement d'une lampe à incandescence étant largement translatée vers le rouge, il est probable que l'énergie rayonnante réelle reçue en simulation soit plus élevée que celle donnée à l'enregistreur ce qui accroît fictivement le rendement calculé.

Observons cependant que les dimensions de chaque capteur $(L=30 \mathrm{~cm}, l=25 \mathrm{~cm})$ correspondent à une surface de $0,075 \mathrm{~m}^{2}$ et que dans ces conditions les fuites thermiques sont très largement supérieures à celles de l'appareil réel qui sera formé de 2 récepteurs de $2 \mathrm{~m}^{2}$ chacun. Nous pouvons donc espérer réduire considérablement les pertes, atteindre une température de stagnation plus élevée et augmenter sensiblement le rendement énergétique du capteur. En effet, les fuites thermiques intéressent essentiellement la surface du capteur. Or les résultats actuels sont relatifs à un capteur de surface totale de $0,36 \mathrm{~m}^{2}$ contenant $0,3 \mathrm{~kg}$ de substance active. Le capteur que nous réalisons pour la réfrigération solaire a une surface totale de $5,2 \mathrm{~m}^{2}$ pour $10 \mathrm{~kg}$ de $\mathrm{CaCl}_{2}$. Ainsi le rapport $\mathrm{m} / \mathrm{s}$ passe de 0,83 à 1,92 . Pour une masse donnée la surface de déperdition est donc 2 fois plus faible dans le second cas (grand capteur) que dans le premier (petit capteur). Nous voulons disposer à l'évaporateur de 1200 frigories nettes pour $4 \mathrm{~m}^{2}$ de captage. Si nous supposons une moyenne journalière de $5 \mathrm{kWh}$ reçus par $\mathrm{m}^{2}$ soit $20 \mathrm{kWh}$ d'énergie solaire pour le capteur complet, le rendement final sera :

$$
\frac{12 \times 4,18}{20 \times 36}=\frac{50,16}{720}=7 \%
$$

ce qui semble une limite supérieure de ce qu'il est possible d'espérer car comme pour l'effet photovoltaíque les $10 \%$ atteints par les cellules au silicium correspondent à air masse 1 , soleil au zénith. Les $5 \mathrm{kWh}$ moyens correspondent à un rayonnement global où le direct peut être fortement incliné sur le plan récepteur. On ne peut pratiquement compter, en ciel clair, que sur une insolation efficace de $9 \mathrm{~h}$ à $16 \mathrm{~h}$ soit $6 \mathrm{~h}$ utilisables. Nos études antérieures ont rarement dépassé $3 \%$. L'appareil réel en voie d'achèvement, nous dira s'il est permis d'espérer davantage. La limite annoncée paraît faible mais interviennent la brièveté de l'insolation efficace même en admettant un ciel clair, la lenteur de l'échauffement de l'adsorbat, la valeur relativement basse de la vitesse de dissociation des ammoniacates, l'existence d'un seul cycle en $24 \mathrm{~h}$, toutes conditions qui contraignent l'expérimentateur à s'adapter au régime d'irradiation incidente. C'est le soleil qui impose son rythme et son apport d'énergie.

\section{Lieu d'expérience :}

\section{Nancy.}

Latitude $48^{\circ} 41^{\prime} 14^{\prime \prime} \mathrm{N}$.

Longitude $06^{\circ} 13^{\prime} 19^{\prime \prime} \mathrm{E}$.

Capteur fixe normal au plan méridien incliné vers le sud.

La normale à son plan ne s'écarte jamais plus de 12 degrés des rayons solaires lorsque le soleil passe au midi vrai. La pente des capteurs ne peut être modifiée que 4 fois par an.

\section{Bibliographie}

[1] Flechon, J., Martin, G., Toure, I., C. R. Hebd. Séan. Acad. Sci. 280B (1975) 211.

[2] Flechon, J., C. R. Hebd. Séan. Acad. Sci. 283B (1976) 127.

[3] Flechon, J., Revue Phys. Appl. 11 (1979) 743-50.
[4] Flechon, J. et Machizaud, F., Revue Phys. Appl. 14 (1979) 97-105.

[5] Flechon, J. et Machizaud, F., Power Sources (à paraître). 